\title{
DIALÉCTICA Y ANALÍTICA: EL CONOCIMIENTO DE LOS PRIMEROS PRINCIPIOS EN ARISTÓTELES
}

\author{
Ana Isabel HERNÁNDEZ NARANJO \\ Universidad Complutense de Madrid
}

Al comienzo del Libro E de la Metafisica escribe Aristóteles: «Toda ciencia basada en la razón o que participa en algo del razonamiento versa sobre causas y principios (archai), ora más rigurosos ora más simples" ${ }^{1}$.

Ante esta afirmación, son dos las cuestiones que inmediatamente se nos plantean: 1. ¿Qué son esas causas y principios?; y 2. ¿Por qué toda ciencia trata sobre ellos?

1. Para responder a esta primera pregunta y esclarecer la naturaleza propia de las causas y de los principios de la ciencia, debemos atender a la concepción aristotélica de la misma.

Ciencia como epistêmê, es decir, como conocimiento en sentido estricto, es aquel razonamiento (sillogismós) por el cual se alcanza el saber. Tal razonamiento, el razonamiento cientifico, no es sino la demostración (apodeixis) ${ }^{2}$. Así que el conocimiento en sentido auténtico, por el cual puede considerarse que, en verdad, se sabe, es concebido por Aristóteles como ciencia demostrativa (epistêmê apodeiktiké).

La estructura de un razonamiento consiste en sentar ciertas cosas y concluir, de un modo necesario a partir de ellas, algo distinto de las mismas ${ }^{3}$. Cuando se trata del razonamiento científico, lo establecido al comienzo y de donde parte ese algo distinto, que en este caso es el conocimiento en sentido propio, recibe

1 Cfr., 1 1025b7-18. La cursiva es nuestra.

2 A. Seg., I, 2 71b19-20.

3 Top., I, 1 100a25-27.

ENDOXA: Series Filosbficas, $n .{ }^{\circ} 10,1998, p p .185-198$. UNED, Madrid 
la denominación de "premisas" ${ }^{4}$ (protasis). Pues bien, los principios y las causas por los cuales nos preguntamos, funcionan, en un sentido ${ }^{5}$, como premisas de la demostración. "Si, pues, el saber es como estipulamos, es necesario también que la ciencia demostrativa se base en cosas verdaderas, primeras, inmediatas, más conocidas, anteriores y causales. En efecto, razonamiento lo habrá también sin esas cosas, pero demostración no: pues no producirá ciencia” ${ }^{6}$. De modo que los principios o premisas a partir de los cuales la demostración científica concluye y obtiene conocimiento en sentido estricto, se caracterizan por ser: verdaderos, porque no es posible saber lo que no es 7 ; primeros en sí mismos, pues son indemostrables, es decir, no pueden ni necesitan ser probados, dado que son ellos la base de toda demostración ${ }^{8}$; inmediatos y primitivos, precisamente por lo último, es decir, porque respecto de ellos no hay una demostración anterior que permita encontrarlos 9 ; más conocidos, porque son cognoscibles de suyo o por naturaleza, es decir, dignos de credibilidad por sí mismos ${ }^{10} \mathrm{y}$, por lo tanto, no necesitan nada para justificarse, siendo precisamente por ello por lo que no son sólo más conocidos, sino, además, mejor conocidos que la conclusión conocida a través de ellos ${ }^{11}$; anteriores por ser causales ${ }^{12}$; y causales, porque sólo se sabe cuando se conoce la causa ${ }^{13}$.

A propósito de esta caracterización aristotélica de los primeros principios de la ciencia demostrativa, son múltiples los debates surgidos entre los intérpretes. Wieland 14 , por ejemplo, concibe los principios al modo de los conceptos de reflexión kantianos, como conceptos no sustantivos, referidos a las estructuras formales del lenguaje, útiles para hacer distinciones y encontrar los argumentos apropiados en cada caso. Otra de las interpretaciones más re-

4 Meta., $\Delta, 11012 \mathrm{~b} 13-17$.

5 Más adelante veremos cómo Aristóteles considera que sólo se posee ciencia de algo cuando se conocen sus causas, o principios, que es como en rigor debe entenderse la aserción con la que comenzamos, y cuya relación con lo que ahora exponemos, tendremos ocasión de establecer, completando así su verdadero significado.

6 A. Seg., I, 2 71b20-25. La cursiva es nuestra.

7 Op. cit., 71b26. Ver también: Tóp., I, 1 100a27-30.

8 A. Seg., I, 2 71b27-28, 72a15-16.

9 Op. cit., 72a6-8.

10 Tóp., I, 1 100b1-22.

11 A. Seg., I, 2 72a27-33.

12 Op. cit., $71 \mathrm{~b} 31$.

13 Op. cit., $71 \mathrm{~b} 30$.

14 Cfr. "Aristotle's Physics and the Problem of Inquiry into Principlesw, Articles on Aristothe, p. 136. 
levantes es la de Irwin ${ }^{15}$, que propone concebirlos como primeros principios en un doble sentido: primeros principios objetivos o reales y proposicionales, captados ambos al mismo tiempo. Los principios objetivos son cosas mismas existentes (tales como los cuatro elementos) y no entidades lingüísticas o psicológicas, que causan otras cosas o hechos externos independientes de nuestras creencias acerca de ellos, así que describen una realidad objetiva; este carácter suyo es además el apropiado para que deban ser conocidos por naturaleza. Los principios proposicionales son secundarios, porque se definen por coincidir o concordar con los principios reales, de modo que su verdad está determinada por su correspondencia con los primeros principios no proposicionales $\mathrm{u}$ objetivos; cuando son captados son conocidos para nosotros, y primeros y mejor conocidos por naturaleza en tanto que mencionan las cosas primeras y mejor conocidas.

2. La segunda cuestión que nos planteábamos hace un momento, acerca de la exigencia del conocimiento de los principios o causas como condición del saber en tanto que epistêmê, resulta clara una vez abordada la naturaleza de dichos principios: se trata de la exigencia de un comienzo del conocer. En efecto, puesto que toda demostración parte de unas determinadas premisas, si ha de ser posible la ciencia demostrativa, es entonces imposible una regresión bien al infinito, bien circular, por intentar en cada caso demostrar a su vez la verdad de las premisas ${ }^{16}$, así que es necesario establecer unas premisas absolutamente primeras, esto es, indemostrables, que, como hemos visto, es justo lo que caracteriza a los primeros principios de la ciencia ${ }^{17}$. Esta condición de un comienzo indemostrable, sobre el que fundar todo conocimiento demostrativo, se traduce, eo ipso, en la condición de finitud del conocimiento, es decir, de considerar la ciencia como un conjunto finito de conocimientos establecidos por demostración ${ }^{18}$.

Si lo que define a la epistêmê como saber de los principios o causas de aquello que conoce, se nos desvela como saber fundamentado en principios verdaderos, primeros, inmediatos, más conocidos, anteriores y causales, en el sentido arriba explicado, respecto de lo concluido a partir de ellos, es entonces en

15 Cfr. Aristotle's first principles, p. 3-4.

16 Op. cit., 3 72b25-29; II, 3 90b24-27. Ver también: Meta., Г, 4 1006a6-10.

17 Tóp., VIII, 3 158a36-37.

18 A. Seg., 1, 22 84a9-11, 84a30-34. 
virtud de tales principios, como se conoce por demostración todo lo que constituye objeto de conocimiento en sentido estricto ${ }^{19}$.

Mas, ¿cómo se conocen los primeros principios?

\section{Los primeros principios y las premisas de la demostración silogística}

La forma estricta que Aristóteles reserva para el conocimiento científico, en tanto que estructurado demostrativamente como sabemos, es la del argumento deductivo (lógoi syllogismón) o deducción concluyente y lógicamente válida: el silogismo. Así que la demostración científica propiamente dicha, es un tipo de silogismo llamado demostrativo (epistemonikós), y, por eso, el modo por el que se depara auténtico conocimiento es por demostración silogística. Pues bien, la forma que ésta presenta se articula en torno a tres elementos, a saber ${ }^{20}$ : la conclusión, que es lo que se demuestra, las estimaciones o axiomas (axiómata) supuestos, a partir de los cuales se demuestra, y el género o sujeto al que se refiere aquello que se demuestra, es decir, el objeto propio de conocimiento científico. Puesto que los primeros principios de la ciencia demostrativa, decíamos, constituyen las premisas de la demostración, es decir, son los principios demostrativos de la ciencia, no pueden corresponder sino a los axiomas que se encuentran al comienzo de la demostración, y a partir de los cuales se establece la conclusión, esto es, el conocimiento en tanto que epistêmê. Aristóteles distingue dos tipos de axiomas ${ }^{21}$ : los comunes a todas las ciencias y los propios ${ }^{22}$ de cada

19 Aristóteles expresa esta anterioridad de los principios en el orden del conocimiento, en los términos siguientes: "Y lo más cognoscible son los primeros principios y las causas (pues mediante ellos y a partir de ellos se conocen las demás cosas, no ellos a través de lo que les está sujeto)" (Meta., 2 98b2-3).

20 A. Seg., I, 7 75a40-75b2. Ver también: Meta., 2 997a6-12.

21 A. Seg., I, $1076 \mathrm{a} 31-43$.

22 Los axiomas especiales de cada una de las ciencias son (A. Seg., 1, 2 72a18-24) las whipótesis" (bypotheses) y las "definiciones" (horismot). Las primeras son tesis que informan si se da o no el género de cosas que constituye el dominio propio de la ciencia en cuestión. Las segundas afirman qué son de suyo las cosas de dicho género, exponiendo sus atributos esenciales.

Reparemos en este momento en que el objeto propio de la ciencia, tal y como lo concibe estrictamente el Estagirita, es, no tanto el género, según acabamos de decir, como un solo género (A. Seg., I, 28 87a38-87b4; Meta., K, 7 1063b36-1064a9). Cada ciencia, en efecto, versa sobre un único género. De ahí que, a parte de los principios comunes a todas las ciencias, cada una de 
ciencia. Ambos son las premisas de la ciencia demostrativa, porque son al mismo tiempo la causa (aitiat) propia de lo conluido por deducción silogística; y esto porque, según el famoso dictum aristotélico, la ciencia es el conocimiento de la causa por la cual es aquello que se conoce ${ }^{23}$. Así se explica ahora que en aquel breve texto que recogimos al comenzar estas páginas, Aristóteles hiciese equivaler los principios y las causas, como definidores del saber ${ }^{24}$.

Saber la causa de algo es saber la razón o el porqué ( $t \dot{d}$ diá $t \hat{i})^{25}$ de que ese algo sea lo que es, según cuatro sentidos posibles, que son justamente los cuatro tipos en que divide Aristóteles las causas 26: la causa material o el "sujeto" (bypokeimenon), la formal o "substancia» (ousía) y "esencia" (to tí ên eînai), la eficiente o "de donde procede el principio del movimiento" y la final o "fin de cualquier movimiento». El conocimiento del porqué (epistêmê tou di'hoti) que proporcionan los primeros principios, en tanto que premisas que aducen las causas propias de aquello de lo que se pretende tener ciencia, es el conocimiento peculiar de la explicación. De modo que la ciencia demostrativa o epistêmê consiste en la explicación de lo concluido a partir del conocimiento de su causa, y los primeros principios demostrativos, puesto que manifiestan esa causa, son en sí mimos explicativos.

Ahora bien, cuando Aristóteles define la ciencia como el conocimiento de la causa por la que algo es, añade: "y no cabe que sea de otra manera» 27 . Puesto que lo que no puede ser de otro modo que como es, es necesario ${ }^{28}$, lo susceptible de explicación científica por principios, es lo necesario, y no ${ }^{29}$ lo acci-

ellas posea unos principios especiales, a partir de los cuales concluye el conocimiento del género propio que circunscribe como su objeto de conocimiento. Y, puesto que existen pluralidad de géneros, también son plurales tanto los principios, como las ciencias de las cuales aquéllos son principios de demostración. Según esto, inmediatamente se constata, como insistentemente hace Aristóteles, la imposibilidad de reducir todos los principios de las ciencias a un único principio del cual se deriven todos los demás. Y es bajo esta consideración como debe entenderse la afirmación aristotélica de la autonomía de las ciencias particulares ( $A$. Seg., I, 32 88a19-37).

23 Meta., A, 1981 a25-30, 2 982a10-14, 982a28-982b9.

24 Ver nota 5.

25 A. Seg., I, $675 \mathrm{a} 35,78 \mathrm{a} 26$.

26 Meta., $\Delta, 3$ 983a24-35. Ver también: Cfr., 2 1013a24-1013b5.

Las cuatro clases de causas se identifican con la función lógico-deductiva de lo que Aristóteles denomina "término medion de la demostración en sus Analíricos ( $A$. Seg., II, 2 89b38-90a7, 3 90a35, 6 75a11-17; 11 94a20-24).

27 A. Seg., I, $271 \mathrm{~b} 12$.

28 Meta., $\Delta, 5$ 1015a34-35. Ver también: $A$. Seg., I, 4 73a21-25.

29 Meta., E, 2 1027a20-27. Ver también: Cfr., K, 8 1064b30-1065a5. 
dental o causal, que ni es siempre, ni generalmente ${ }^{30}$. Resumiendo, podemos entonces afirmar que la ciencia es la demostración silogística de por qué un género de cosas es como es y no puede ser de otra manera. Con esto, demos ahora un paso más para escuchar en el carácter de necesidad, exigido como condición definidora del objeto propio de conocimiento, los ecos de la concepción platónica de la ciencia: nos referimos a la estabilidad tan requerida por Platón para la constitución específica del saber en tanto que epitêmê, frente a la inestabilidad, agitación y movimiento qua acompañan, de un modo también específico a la doxa. Por eso no es extraño encontrar a su discípulo escribiendo, "de suerte que, si ha de haber ciencia y conocimiento de algo, es preciso que haya aparte de las sensibles, otras naturalezas estables, pues de las cosas que fluyen no hay ciencia" ${ }^{31}$. La pregunta es ahora cuáles son esas naturalezas. Si estamos en lo cierto, y el objeto de conocimiento es lo estable por ser necesario, éste, y, por ende, dichas naturalezas, son lo universal ${ }^{32}$ (kathólou). Como, por otro lado, lo universal, según Aristóteles, indica la causa ${ }^{33}$ y es mejor conocido ${ }^{34}$ que lo singular que ello mismo explica, bien puede considerarse que se trata de lo que define a los primeros principios en cuanto tales. Y si es el conocimiento de éstos lo que perseguimos "está claro, entonces, que nosotros, necesariamente, hemos de conocer por inducción (epagoge), pues así "es como" la sensación produce "en nosotros" lo universal» 35 , estabilizando lo que de universal hay en la experiencia (empeiria).

Ahora, ¿cómo procede e interviene la inducción en el conocimiento de los primeros principios?

\section{La inducción desde los phainomena}

La inducción "es el camino desde las cosas singulares hasta lo universal» 36 , pues esto último se da, necesariamente, en los singulares y no separado de

Op. cit., $21026 \mathrm{~b} 33$.

Op. cit., 4 1078b15-17. La cursiva es nuestra.

A. Seg., I, $3388 \mathrm{~b} 30-33$.

Op. cit., $3188 \mathrm{a} 5$.

34 Ver también: Op. cit., 87a38; Tóp., VIII, 14 164a7-11; Meta., B, 6 1003a15, $\Delta, 11$ 1018b31-34.

35 A. Seg., II, 19 100b3-5.

36 Top., I, 12 105a12-14. 
ellos ${ }^{37}$; consiguientemente, sólo a través de la inducción, que parte de los singulares, es posible "contemplar" (theorêsai) los universales de los que parte la demostración. Por el proceso de inducción se reconoce y comprueba - no se conoce- lo universal, confusamente presente en lo particular, hasta depurarlo y fijarlo de forma clara y distinta, momento en que se constituye plenamente como tal. Aristóteles ha diferenciado en este proceso cuatro estados o disposiciones (héxis) necesarios para la comprobación de lo universal y, con ello, para la adquisición de los principios ${ }^{38}$ : (1) Intervención de la percepción o sensación (aisthésis) como facultad (dynamis) con la cual distinguir y discriminar los casos particulares; (2) retención en la memoria de lo que es así percibido por los sentidos; (3) surgimiento de una experiencia unitaria por la repetida acumulación de lo retenido en la memoria; y (4) producción del universal, inicialmente indeterminado e indiferenciado en lo percibido en cada uno de los casos singulares, en tanto que "concepto" (lógos), podríamos añadir, explicativo.

Lo percibido, a partir de lo cual se inica el proceso de inducción, son los «fenómenos" (phainomena), considerados por Aristóteles, como bien ha señalado Owen ${ }^{39}$, en un doble sentido: lo éndoxa y legomena, por un lado, y lo phainomenon propiamente perceptuah por otro. Este último se refiere sin más a los puros datos empíricos, captados por la experiencia sensible. Los dos primeros son los que constituyen propiamente el punto de partida del proceso de inducción en el conocimiento de los principios, en la medida en que ambos representan el conjunto de creencias (hypolepseon) comúnmente aceptadas en una comunidad determinada, y es a través del proceso de inducción como se explicitan los términos conceptuales presupuestos en ellas de un modo vago e indiferenciado ${ }^{40}$. Así que, partiendo de lo éndoxa y lo legomena, que son, por tanto, lo más cognoscible para nosotros, nos encaminamos hacia el conocimiento de los primeros principios, que son lo menos cognoscible para nosotros, pero más y mejor congnoscibles por naturaleza y en sí mismos, hasta hacerlos, por induc-

37 A. Seg., I, 4 73b27-30; Meta., Z, 16 1040b26-27.

38 A. Seg., Il, $1999 \mathrm{~b} 32-100 \mathrm{~b} 5$.

39 Cfr. "Tithenai ta Phainomena", Articles on Aristotle, pp. 114-118.

40 IRWIN (Cfr. Aristotle's first principles, pp. 30-31) cuestiona que pueda diferenciarse tajantemente entre las evidencias propiamente empíricas por una parte, y las creencias comunes por otra, al considerar que ambas pueden constituir el comienzo de una investigación, en tanto que las creencias comunes no perceptuales pueden surgir, y surgen, a partir de las apariencias empíricas. 
ción, cognoscibles también para nosotros ${ }^{41}$, al explicitar lo universal conceptualmente implícito en aquellos phainomena credenciales.

Pero ¿cómo tiene lugar ese encaminarse?

\section{La dialéctica hacia los primeros principios}

Tal sería la respuesta de Aristóteles a nuestra pregunta: la dialéctica (dialektike); pues "en efecto, al ser adecuada para examinar "cualquier cosa", abre camino a los principios de todos los métodos" ${ }^{42}$, es decir, a los principios comunes (koinat archai) a toda forma de razonamiento, a través de las «cosas plausibles» ${ }^{43}$ (éndoxon mâllon), que no son sino las creencias, verdaderas o falsas, sostenidas en común por una comunidad particular.

De los tres usos posibles que pueden hacerse de la dialéctica, en tanto que técnica de argumentación a través de preguntas y respuestas, el que aquí nos interesa, por encaminar hacia los primeros principios, y al que los intérpretes suelen referirse como uso constructivo de la dialéctica, es el considerado por el Estagirita como eficaz "para los conocimientos en filosofia", y que se apoya en los otros dos, a saber, los que son últiles "para ejercitarse" y "para las conversaciones) ${ }^{44}$. Ciertamente, en virtud de este uso, que se mueve en un nivel preteórico, el dialéctico "examina» la validez de las creencias corrientes, generalmente aceptadas por su comunidad, y transmitidas por la tradición histórica precedente, descubriendo, de acuerdo con las reglas de la silogística, las falsedades, contradicciones, aporías y paradojas conceptuales encerradas en ellas, hasta establecer, al término del examen, aquellas que, de entre todas, revisten un grado de validez suficiente para constituirse en los primeros principios de las ciencias. Así que, es a partir del análisis dialéctico de las creencias dadas históricamente, y de las tesis u opiniones comúnmente compartidas en una comunidad, como se accede el conocimiento de los principios demostrativos, y por lo que, escribe Artistóteles, "toda enseñanza y todo aprendizaje por el pensamiento se producen a partir de un conocimiento preexistente» ${ }^{45}$. Dicho conocimiento, resul-

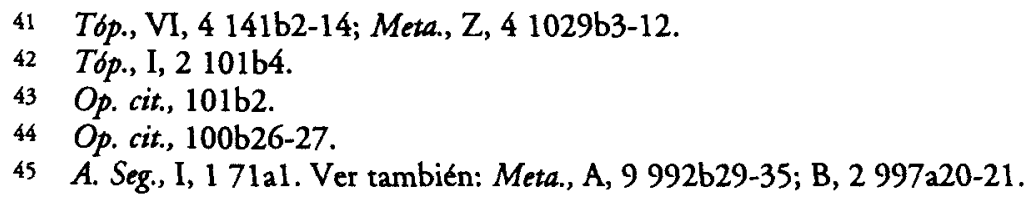


ta claro, está constituido por lo que arriba llamábamos lo éndoxon y legomenon. Éndoxon, en efecto, significa "generalmente aceptado", pero no sólo esto, pues también equivale a "probable" o "plausible", como hace un momento vimos usar a Aristóteles. La relación entre ambos significados de lo éndoxa supone que las creencias compartidas poseen diferentes grados de plausibilidad, en función de su aceptación por los miembros más reputados y distinguidos de la comunidad, de modo que uson cosas plausibles las que parecen bien a todos, o a la mayoría (to hôs epi to polu), o a los sabios, y, entre estos últimos, a todos, o a la mayoría, o a los más conocidos y reputados" 46.

Ahora bien, lo que inmediatamente pone de manifiesto el hecho de que los primeros principios se encuentren dialécticamente desde lo éndoxa y legomena, habida cuenta el valor de plausibilidad inherente a los últimos, es el carácter radicalmente problemático del estatuto epistemológico de dichos principios, pues resultan ser universales, y, al mismo tiempo, plausibles o verosímiles, lo cual les aleja inevitablemente de la necesidad y objetividad propia de la ciencia demostrativa, cuyas premisas, como decíamos, están encarnadas por ellos, así como niega precisamente aquella primera nota con la que, si recordamos, Aristóteles caracterizaba los primeros principios, y según la cual éstos son verdaderos.

Irwin ha señalado esta índole esencialmente problemática de los primeros principios de las ciencias, debido a su valor dialéctico, del siguiente modo: la dialéctica, al argumentar de acuerdo con las creencias, no es capaz de proporcionar verdad, sino sólo una coherencia lógica interna a dichas creencias, que no es suficiente para justificar, por sí misma, la verdad objetiva de los primeros principios. Como vía posible de solución a este problema, Irwin propone el método de la "strong dialectio" ${ }^{47}$, que vendría a ser el empleo de la técnica dialéctica en orden a aislar, según vimos al presentar su uso constructivo, un subconjunto de lo éndoxa especialmente relevante por su corrección, y tal que sirva por ello como "nuevo comienzo", dice Irwin, de la ciencia demostrativa, libre de toda disputa, más próximo a la verdad y, por consiguiente, más objetivo. Con todo, la dificultad persiste, pues la strong dialectic no pasa de ser una estrategia hermeneútica que permite la elección de un nuevo comienzo de la ciencia, en virtud de las necesidades mismas del discurso argumentativo y, por lo tanto, en exclusiva referencia a las prácticas discursivas en el seno del contexto comuni-

46 Tóp., I, 1 100b22-24. Ver también: 14 105a35-105b1.

47 Cfr. Aristotle's first principles, p. 21-25, 43-45. 
cativo de la comunidad que comparte lo éndoxa. Por eso termina Irwin concluyendo que la dialéctica, ciertamente, es el camino necesario hacia los primeros principios, pero en modo alguno el método por el que, de hecho, se alcancen. Y es por salvar esta debilidad de la dialéctica, por lo que Aristóteles necesita, a juicio de Irwin ${ }^{48}$, apelar a una intuición (noûs) capaz, ésta sí, de justificar por ella misma la verdad objetiva de los primeros principios. La técnica dialéctica, en tanto que camino hacia los principios, sólo conseguiría entonces generar la héxis o condición epistémica adecuada para acceder, por intuición, al conocimiento de los principios demostrativos de las ciencias, pero no sería en sí misma su captación. Por ello, afirma Irwin, inducción y dialéctica pueden considerarse "estimulantes" y "ocasiones» para la intuición, pero no pueden ir más allá de la creencia y la de la plausibilidad; sólo la intuición puede justificar la verdad de los primeros principios una vez alcanzados ${ }^{49}$.

\section{La intuición de los primeros principios}

Con la intuición llegamos a la héxis propiamente peculiar del conocimiento de los principios. En esta disposición epistémica los principios, como conceptos universales en última instancia, son captados de un modo puramente intelectual e intuitivo ${ }^{50}$, siendo así que son conocidos en sí mismos, y no es

48 Op. cit., pp. 136 ss.

49 Asimismo, en la necesidad de recurrir a la intuición, como el modo propio de acceder al conocimiento de los primeros principios, encuentra IRWIN (op. cit., pp. 139-140) la confirmación de la autonomía de las diferentes ciencias, tan defendida por Aristóteles, pues al ser captados por intuición, los principios se muestran como verdaderos y primarios en sí mismos, no derivados de nada más $\mathrm{y}$, consiguientemente, tornan innecesaria una apelación ulterior a la coherencia lógica o a alguna ciencia más alta, que proporcione la base de todas las ciencias particulares (ver nota 22).

50 Así por ejemplo, cuando aprehendemos por intuición el principio de contradicción captamos la imposibilidad de que "la misma cosa sea y no sea en un solo y mismo tiempo" (Meta., $\mathrm{K}, 5 \mathrm{1061b38}$ ). Ahora bien, ¿significa esto que imponemos nuestra propia constitución cognoscitiva a lo que es, en la medida en que pretendemos conocerlo, dado que nuestra constitución cognoscitiva, en tanto que racional, no nos hace posible concebir que una misma cosa sea, al mismo tiempo y en el mismo sentido, no sea, de manera que nuestro conocimiento de lo que es, es, ante todo y en primer lugar, el conocimiento que nuestra racionalidad nos permite alcanzar, pero sin garantizarnos que es realmente como lo conocemos, es decir, tal y como nuestra racionalidad cognoscitiva nos da a conocer que es, o significa, a la inversa, que lo que es impone su constitución ontológica, o su modo propio de ser, a nuestra estructura racional de conocimiento, y así, es porque es realmente imposible que la misma cosa sea y no sea en un solo y mismo tiem- 
necesaria ninguna posterior justificación del estatuto de verdad que los define en tanto que principios de las ciencias, pues una vez alcanzados, fijan las premisas que constituyen el comienzo a partir del cual discurre por deducción silogística cada una de las ciencias particulares 51 .

No obstante, la relación que guardan intuición y dialéctica, respecto del conocimiento de los primeros principios, sigue siendo problemática: ¿cómo conciliar la plausibilidad de los principios, por ser alcanzados dialécticamente desde lo éndoxa y lo legomena, con la verdad que les debiera caracterizar, por ser aprehendidos intuitivamente?, ¿cómo pueden ser los principios plausibles y verdaderos al mismo tiempo?, ¿existe una discontinuidad epistémica insalvable entre lo éndoxa y la verdad?

$\mathrm{Si}$, como al principio, volvemos a preguntarnos ahora por la concepción aristotélica del conocimiento en sentido estricto, afirmaremos que éste es el resultado obtenido por demostración o deducción silogistica a partir de unas premisas que no son sino los primeros principios universales, y dialécticamente verdaderos. Este extrańo valor de verdad dialéctica propia de los principios de las ciencias, dado el modo en que llegan a sernos conocidos, resulta aún más problemático, pero no por ello menos rico, si no olvidamos que el conocimiento de tales principios está determinado, en última instancia, por la verdad misma de las cosas, dice Aristóteles ${ }^{52}$. Esto, en principio, significa suponer que el orden propio del conocimiento está determinado por un orden objetivo de ser, de un modo tal que, en el caso especial del conocimiento de los primeros principios, si éstos, como hemos visto, son lo más y mejor cognoscible de suyo y, por lo tanto, son epistemológicamente anteriores respecto del conocimiento de las cosas deducido de ellos, deben ser, por eso mismo, ontológicamente anteriores o primeros respecto del ser de las cosas por ellos causadas, pues como sabemos, los principios son también causas. En consecuencia, parece que los primeros

po, por lo que captamos y decimos conocer que ues imposible que los contrarios pertenezcan a la vez a un mismo sujeton? (Cfr. $\Gamma, 3$ 1005a28) ¿Sería esta segunda posibilidad la que suscribiría Aristóteles?

51 "(...) en efecto -escribe el Estagirita- llamo intuición al principio de la ciencia" ( $A$. Seg., I, 33 88b38. Ver también: II, 19 100b10-15).

52 Meta., A, $3984 \mathrm{~b} 10$. 
principios son tanto los principios constituyentes desde el punto de vista ontológico, como los principios demostrativos y, por ello, explicativos, desde el punto de vista epistemológico, del ser y del conocimiento propios de las cosas derivadas de ellos. Sólo mediante una estricta defensa metafísica del realismo y epistemológica del fundamentalismo, pueden justificare estas tesis, del mismo modo que sólo bajo una concepción de la verdad como correspondencia con una realidad objetiva, puede entenderse, en último término, esa prioridad ontológica y epistemológica de los primeros principios. Es en estos presupuestos filosóficos últimos donde reside la verdadera razón por la que, siguiendo a Irwin, considerábamos que la mera coherencia interna de las creencias no garantiza la verdad de los principios alcanzados por el examen dialéctico de las mismas, de manera que para justificar definitivamente su verdad, sería necesario determinar la correspondencia efectiva entre los principios y la objetividad de lo real 53 .

Wieland ${ }^{54}$, en cambio, niega en la metafísica de Aristóteles la existencia de una esfera objetiva del ser, e independiente del modo en que el discurso cognoscitivo y la argumentación hablan de ella: ser y ser dicho son intercambiables, puesto que en el uso del verbo ser se articula una estructura lingüística. Frente a esta interpretación, Aubenque 55 argumenta en favor de una concepción de la verdad como correspondencia en el pensamiento de Aristóteles. Comienza el profesor francés constatando la contraposición existente entre ciertos pasajes de los Libros y de la Metafisica, a propósito de esta cuestión: según el primero, lo verdadero y lo falso son funciones lógicas del juicio, y, por tanto, no están en las cosas ${ }^{56}$, sino más bien en el pensamiento; en el segundo, sin embargo, el enlace en el pensamiento se considera verdadero en tanto en cuanto expresa un enlace en las cosas, es decir, si se piensa, parafraseando a Aristóteles, que lo separado está separado y que lo junto está junto ${ }^{57}$; y termina descubriendo una posible clarificación — no solución- de la dificultad que entraña la compren-

53 Según esto, y atendiendo ahora concretamente a la definición aristotélica de epitêmê como ciencia demostrativa, si la demostración silogística es capaz de explicar por qué ciertas cosas son necesariamente como son, se sigue entonces que la logica, de un modo general, representa la onticidad Pero, ¿por qué la silogistica expresa, con verdad, lo que es necesariamente?, ¿por qué el ser necesario se dice silogisticamente?, ¿por qué el logos del ente necesario es el silogismo?, ¿cuál es, en definitiva, el auténtico fundamento de la correspondencia entre el logos y lo ente?

54 Cfr. «Aristotle's Physics and the Problem of Inquiry into Principles", Articles on Aristothe, p. 133.

55 Cfr. El problema del ser en Aristóteles, pp. 159-162.

56 Cfr. 2 1026a34-1026b3.

57 Cfr. $101051 \mathrm{a} 34-1051 \mathrm{~b} 9$. 
sión de estos pasajes, en lo implicado por la consideración de que «cada cosa tiene verdad en la misma medida en que tiene ser" ${ }^{58}$, pues esto supone que el juicio sólo desvela una relación entre las cosas, que es prioritaria con respecto a aquél. De manera que, conluye Aubenque, la verdad del discurso está siempre dada de antemano en las cosas mismas, que son lo que primordialmente hay, aun cuando, ciertamente, sólo se desvela a través del discurso y sólo se traduce por medio del lenguaje, en los cuales, no obstante, nunca es alcanzada por completo.

Que cada cosa tenga verdad en la misma medida en que tenga ser, no significa sino que todo lo que es, por el mero hecho de ser, es ya verdadero y, por lo tanto, susceptible de ser dicho con verdad o falsedad, tesis ésta que confirma el realismo metafísico y el fundamentalismo epistemológico en los cuales encardinábamos hace un momento la concepción aristotélica del conocimiento en sentido estricto. Pues bien, en la medida en que los primeros principios satisfacen, en tanto que premisas, la exigencia de un comienzo de la ciencia demostrativa, tal y como es caracterizada por estos dos presupuestos filosóficos, dichos principios son, por su prioridad epistemológica y ontológica, la expresión más genuina de los mismos; así como su conocimiento, la manifestación última de la estrecha relación existente entre silogística o analítica y dialéctica en la filosofía de Aristóteles. La analítica gira en torno a la deducción y demostración silogistica propia del proceder de las ciencias, a partir de unas premisas constituidas por los primeros principios, moviéndose, por tanto, en el ámbito de la explicación y de la enseñanza del saber. El conocimiento de los primeros principios, gira, antes bien, alrededor de la dialéctica, la cual no parte de ellos, sino que argumenta en su búsqueda (têtêsis) 59, moviéndose, por consiguiente, en el dominio propio de la investigación; por eso, la "ciencia que se busca" 60 es la ciencia de los primeros principios.

58 Op. cit., $\alpha, 1993630$.

59 Op. cit., K, $71063 \mathrm{~b} 36$.

60 Ver, por ejemplo: Op. cit., A, 2 982b8-9. 


\section{Bibliografía}

\section{Obras fuente}

ARISTÓteles: Analíticos Segundos (traducción de M. Candel Sanmartín), Editorial Gredos, Madrid, 1988.

- Metafisica (traducción de V. García Yebra), Editorial Gredos, Madrid, 1982.

- Tópicos (traducción de M. Candel Sanmartín), Editorial Gredos, Madrid, 1988.

\section{Bibliografia secundaria}

Barnes, J.: «Aristotle's Theory of Demonstration", Articles on Aristotle, Edited by J. Barnes, Malcom Schofield, Richard Sorabji, Duckworth, 1975.

Hankinson, R. J.: "Philosophy of Science", The Cambridge Companion to Aristotle, Edited by J. Barnes, Cambridge University Press, 1995.

- "Science», The Cambridge Companion to Aristotle, ed. por J. Barnes, Cambridge University Press, 1995.

IRWIN, T. H.: Aristotle's first principles, Clanderon Press, Oxford University Press, 1988.

MAS TORres, S.: “El concepto aristotélico de Ciencia y la Dialéctica como práctica», Endoxa. Series Filosóficas, n. ${ }^{\circ}$ 4, pp. 83-98, UNED, Madrid.

OWEN, G. E. L.: "Tithenai ta Phainomena", Articles on Aristotle, ed. por J. Barnes, Malcom Schofield, Richard Sorabji, Duckworth, 1975.

VeGA, L.: "Tà éndoxa: argumentación y plausibilidad", Éndoxa: Series Filosóficas, n. ${ }^{\circ}$ 1, pp. 5-19, UNED, Madrid.

- La trama de la demostración (Los griegos y la razón tejedora de pruebas), Alianza Universiad, Madrid, 1990.

WeIL, E.: "The Place of Logic in Aristotle's Thought", Articles on Aristotle, ed. por J. Barnes, Malcom Schofield, Richard Sorabji, Duckworth, 1975.

Wieland, W.: «Aristotle's Physics and the Problem of Inquiry into Principles", Articles on Aristotle, ed. por J. Barnes, Malcom Schofield, Richard Sorabji, Duckworth, 1975. 